\title{
PCR for cellular forces
}

A 'mechanically induced catalytic amplification reaction' reads out receptor-mediated forces in cells.

There is a growing appreciation of the importance of mechanical forces in cell biology. Cells rely largely on mechanosensitive proteins to 'feel' their environment. The mechanical forces mediated by such receptors are important in diverse processes such as stem cell differentiation, wound healing and biofilm formation. Molecular forces present a detection challenge, however: not only are they tiny in magnitude (on the order of piconewtons), but they are also transient.

Khalid Salaita of Emory University believes that the mechanobiology field is in need of a

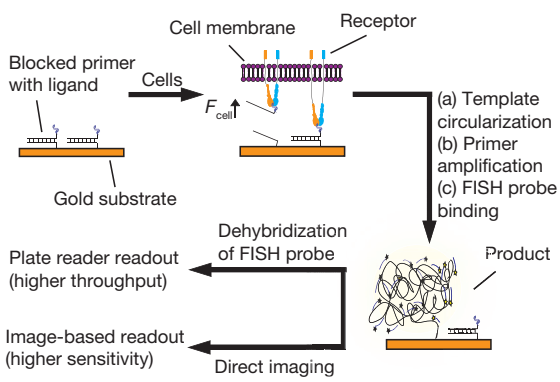

The mechanically induced catalytic amplification reaction. Figure adapted with permission from $\mathrm{Ma}$ et al., 2016. ${ }^{\odot}$ John Wiley and Sons. methodological revolution. "There is a stark disconnect between the importance of mechanics and our understanding of its role in biology," he says. "This is because we don't have the right tools and the right accessible tools to start measuring mechanics in cells in every cell biology lab."

Salaita and his colleagues were inspired by PCR and ELISA, which both rely on catalytic amplification to detect either nucleic acids or proteins, to develop a straightforward approach for measuring mechanical forces mediated by cell surface receptors. They call their method the 'mechanically induced catalytic amplification reaction,' or MCR. This approach converts piconewton forces into a readily detectable and quantifiable

\section{MOLECULAR BIOLOGY}

\section{OF TRIBE AND RNA TAGGING}

\section{Genetic approaches profile RNA-protein interactions.}

RNA molecules often pair with RNA-binding proteins (RBPs) to regulate cellular processes. To understand how such RNA-protein networks function, it is important to know which transcripts are bound by particular proteins in individual cell types.

Cross-linking and immunoprecipitation (CLIP) and its derivatives are powerful in vitro methods for profiling RNA-protein interactions. Recent improvements such as infrared CLIP and enhanced CLIP (on pages 482, 489 and 508 of this issue) allow faster profiling from fewer cells; they yield base-pair resolution of the binding site but still require specific antibodies and tens of thousands of cells as input material, a prohibitive quantity for applications to rare cell types.

Now independent efforts from the groups of Michael Rosbash at Brandeis University and Marvin Wickens from the University of Wisconsin have added a genetic approach to complement CLIP techniques.

The Rosbash team is interested in deciphering the molecular regulation of circadian rhythms, particularly in post-transcriptional gene regulation in clock neurons in flies. When using biochemical methods to determine the RNA-binding profile of proteins involved in circadian biology, the researchers hit a roadblock early on. "The methods that are available were not suitable. You need a reasonable amount of material to get enough signal," says Rosbash. Instead of performing CLIP, they wanted to label the protein-bound RNA directly in a subset of neurons and identify them by sequencing.

The question was how to label the bound RNA. The Rosbash team had previously examined neuron-specific RNA editing involving the deamination of adenosine to inosine, which can be reverse transcribed as a guanosine to locate edited sites. Taking advantage of this process, they fused the catalytic domain of a deaminase (ADAR) to a protein of interest and identified any direct RNA targets by the presence of edited sites (McMahon et al., 2016). They validated their method, called TRIBE (targets of 
fluorescent signal, and it utilizes techniques and equipment that are common in most cell biology labs.

To measure forces mediated by a mechanosensitive receptor on the surface of a cell, MCR utilizes an immobilized DNA duplex modified with a ligand as a mechanically responsive element. This DNA duplex can be designed with a particular tension tolerance; if the force applied by cell surface receptors exceeds the tension tolerance, the duplex will be denatured. The process of denaturation exposes a blocked primer, making it available for amplification by isothermal rolling-circle amplification (RCA). The amplified product is visualized using fluorescence in situ hybridization (FISH) and quantified either by imaging the surface or by releasing the fluorescent oligonucleotides for analysis with a plate reader.

Salaita’s team applied MCR to study mechanical forces mediated by integrin receptors, potentially important drug targets that facilitate cell adhesion and migration, in NIH/3T3 fibroblasts. They designed two DNA duplex elements with different tension tolerances. When they plated the cells on top of these elements and carried out MCR, they observed fluorescent signal levels that were dependent on the tension tolerance. Control experiments verified that MCR was specific for detecting force. They used the approach to measure the effect of the non-muscle myosin II inhibitor blebbistatin on integrin mechanics, and they observed a dose-dependent response that further confirmed that MCR provides a direct readout of force.

Salaita is particularly excited about the prospect of using MCR to screen for drugs that alter cell mechanics. As he explains, high-throughput screening will require adapting the reaction to a glass-bottomed 96-well plate and automating the sample preparation and amplification steps.

Salaita notes that MCR should also be useful for assigning mechanical signatures to cells, for investigating the transient 'force footprint' generated by cell surface receptors, and for studying how different receptors transmit distinct magnitudes of force, especially in cancer. Allison Doerr

RESEARCH PAPERS

Ma, V.P.-Y. et al. Mechanically induced catalytic amplification reaction for readout of receptor-mediated cellular forces. Angew. Chem. Int. Ed. 55, 5488-5492 (2016).

RNA-binding proteins identified by editing), with CLIP data. However, it is not clear how the enzyme selects the adenosine for editing. "The specificity of this enzyme is more than a bit of a mystery," says Rosbash. ADAR needs double-stranded RNA to bind and will thus miss targets that do not form such structures.

The Wickens group selected a different enzyme to mark the protein-bound RNA. For many years, they studied nucleotidyl transferases, and that work unexpectedly led them to discover enzymes that added uridines to the end of transcripts. "It was clear then that, in principle, these could be used in tagging," says Wickens. They fused a poly(U) polymerase (PUP) to the protein of interest and confirmed that a uridine tail was added only to transcripts that bound the protein (Lapointe et al., 2015). Their RNA-tagging approach requires paired-end sequence reads to identify the mRNA and the number of U's added, but it does not require a particular RNA structure. Wickens sees the methods as complementary: "together the two methods track events RNAs undergo in cells, leaving a record of their histories."

One of Rosbash's goals is to apply TRIBE to translation. "That is a frontier for us that we would like to conquer," he says: "which transcripts are actually being translated in small numbers of neurons."

Wickens wants to follow the assembly of large networks in cells and how they are exploited for regulation. "Multiple different proteins bind a single mRNA, and it is unclear whether those proteins collaborate, compete or act independently," he says.

The tagging of RNA will likely not stop with these two enzymes, and Wickens concludes, "The development of new tagging enzymes—like ADAR—should enable multiple proteins to be probed simultaneously, and at the level of individual RNA molecules. The more we have, the better."

\section{Nicole Rusk}

\section{RESEARCH PAPERS}

McMahon, A.C. et al. TRIBE: hijacking an RNA-editing enzyme to identify cell-specific targets of RNA-binding proteins. Cell 165, 1-12 (2016).

Lapointe, C.P. et al. Protein-RNA networks revealed through covalent RNA marks. Nat. Methods 12, 11631170 (2015). 